\title{
Casimir energy of Sierpinski triangles
}

\author{
K. V. Shajesh, ${ }^{1,2, *}$ Prachi Parashar, ${ }^{2, \dagger}$ Inés Cavero-Peláez, ${ }^{3, \$}$ Jerzy Kocik $^{4, \S}$ and Iver Brevik ${ }^{2, \uparrow}$ \\ ${ }^{1}$ Department of Physics, Southern Illinois University-Carbondale, Carbondale, Illinois 62901, USA \\ ${ }^{2}$ Department of Energy and Process Engineering, Norwegian University of Science and Technology, \\ N-7491 Trondheim, Norway \\ ${ }^{3}$ Centro Universitario de la Defensa (CUD), Zaragoza 50090, Spain \\ ${ }^{4}$ Department of Mathematics, Southern Illinois University-Carbondale, Carbondale, Illinois 62901, USA
} (Received 19 September 2017; published 13 November 2017)

Using scaling arguments and the property of self-similarity we derive the Casimir energies of Sierpinski triangles and Sierpinski rectangles. The Hausdorff-Besicovitch dimension (fractal dimension) of the Casimir energy is introduced and the Berry-Weyl conjecture is discussed for these geometries. We propose that for a class of fractals, comprising compartmentalized cavities, it is possible to establish a finite value to the Casimir energy even while the Casimir energy of the individual cavities consists of divergent terms.

DOI: 10.1103/PhysRevD.96.105010

\section{INTRODUCTION}

Weyl's law [1], which was originally discussed for the spectral distribution of the modes allowed inside a Dirichlet cavity, when extended for the Casimir energy per unit length $\mathcal{E}(a)$ of a polygonal cylindrical cavity with a single characteristic scale $a$, in natural units of $\hbar=c=1$, states that

$\mathcal{E}(a)=\frac{b_{c}}{a^{2}}+\lim _{\tau \rightarrow 0} \frac{1}{\tau^{2}}\left[b_{2} A\left(\frac{a}{\tau}\right)+b_{1} P\left(\frac{a}{\tau}\right)+b_{0} C\left(\frac{a}{\tau}\right)\right]$,

where the coefficients of the divergent terms, $A(x), P(x)$, and $C(x)$, scale like the area of the cavity, the perimeter of the cavity, and the corner angles of the cavity, respectively. That is,

$$
A(x) \propto x^{2}, \quad P(x) \propto x^{1}, \quad C(x) \propto x^{0} .
$$

Parameters $b_{c}, b_{2}, b_{1}$, and $b_{0}$ in Eq. (1) are dimensionless constants. The parameter $\tau$ is a temporal point-splitting cutoff parameter introduced in the calculation to regulate the divergences.

Berry conjectured [2], again in the context of spectral distribution, that for fractal cavities the Weyl law maintains the form of Eq. (1) with the only difference that the coefficients of the divergent terms, $A(x), P(x)$, and $C(x)$, scale like the Hausdorff-Besicovitch dimension (fractal dimension) of the area of the cavity, the perimeter of the cavity, and the corner angles of the cavity, respectively. That is,

\footnotetext{
*kvshajesh@gmail.com

prachi.parashar@ntnu.no

*avero@unizar.es

$\S$ jkocik@siu.edu

qiver.h.brevik@ntnu.no
}

$$
A(x) \propto x^{\delta_{2}}, \quad P(x) \propto x^{\delta_{1}}, \quad C(x) \propto x^{\delta_{0}},
$$

where $\delta_{2}$ is the fractal dimension of the area of the cavity, $\delta_{1}$ is the fractal dimension of the perimeter of the cavity, and $\delta_{0}$ is the fractal dimension of the corner angles of the cavity.

It is, then, not a long shot to envision that the Casimir energy per unit length of a fractal cavity need not scale like the inverse square of length. Thus, presuming that the energy scales like $a^{\delta_{c}}$, we can generalize Weyl's law in Eq. (1) as

$\mathcal{E}(a)=b_{c} a^{\delta_{c}}+\lim _{\tau \rightarrow 0} \tau^{\delta_{c}}\left[b_{2} A\left(\frac{a}{\tau}\right)+b_{1} P\left(\frac{a}{\tau}\right)+b_{0} C\left(\frac{a}{\tau}\right)\right]$,

where $\delta_{c}$ is the fractal dimension of the Casimir energy per unit length of the fractal cavity.

The central theme of this paper is to use the scaling arguments and the property of self-similarity introduced in Ref. [3] to derive the Casimir energies of Sierpinski triangles and Sierpinski rectangles. We also introduce a class of fractals for which the energy does not scale as inverse length square, which leads us to introduce a fractal dimension for the Casimir energy. One usually associates fractal dimensions to geometrical quantities like perimeter and area, but being able to introduce fractal dimensions to Casimir energy for a class of fractals directly relates to the conventional wisdom that Casimir energy of cavities, satisfying perfectly conducting boundary conditions (or Dirichlet boundary conditions for scalar fields), is purely geometrical. It should not be very surprising, because energy has been shown to exhibit fractal nature before. For example, the Hofstadter butterfly is a fractal that represents the energy of Bloch electrons in a magnetic field [4].

We consider an equilateral triangle even though most of our discussion holds true for an arbitrary triangle. 
The Casimir energy of an equilateral triangular cylinder on which a scalar field satisfies Dirichlet boundary conditions was calculated exactly, in closed form, in Ref. [5]. This involves the Casimir energy of five cylindrical cross sections, namely an equilateral triangle, a hemiequilateral triangle, an isosceles right triangle, a square, and a rectangle; see Fig. 1. For all five geometries the authors of Ref. [5] have shown that the Casimir energy per unit length obeys the Weyl law in Eq. (1). Using Ref. [5], the Casimir energy per unit length of an equilateral triangle is described by the parameters

$b_{c}=-\frac{1}{72}\left[\frac{\sqrt{3}}{9}\left\{\psi^{(1)}\left(\frac{1}{3}\right)-\psi^{(1)}\left(\frac{2}{3}\right)\right\}-\frac{8}{\pi} \zeta(3)\right]$,

with a numerical value $b_{c} \sim 0.0237188$,

$$
b_{2}=\frac{3 \sqrt{3}}{8 \pi^{2}}, \quad b_{1}=-\frac{3}{8 \pi}, \quad b_{0}=\frac{1}{6 \pi},
$$

of Eq. (1), where $\psi^{(m)}(z)$ is the polygamma function of order $m$ and $\zeta(z)$ is the Riemann zeta function. The above evaluation was achieved using the mode summation method, which presumes that the Casimir energy of a closed Dirichlet cavity is completely determined by the modes in the interior of the cavity alone [5]. This should be
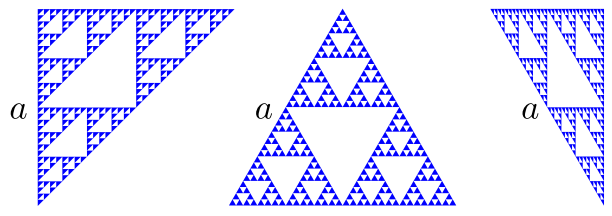

$$
\mathcal{E}_{s}=-\frac{4}{11} \mathcal{E}_{\Delta}
$$

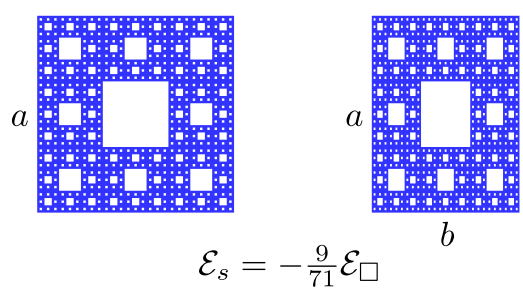

FIG. 1. Gallery of Sierpinski cylinders with Casimir energies per unit length $\mathcal{E}_{s}$ for the five integrable cylinders studied in Ref. [5]. Top row: An isosceles right triangle with the equal sides of length $a$, an equilateral triangle of side length $a$, and a hemiequilateral triangle with length of hypotenuse $a$. Bottom row: A square of side length $a$, and a rectangle of side lengths $a$ and $b$. The Casimir energy per unit length of a Sierpinski triangle is $-4 / 11$ times the Casimir energy per unit length of the respective triangle, $\mathcal{E}_{\Delta}$, and the Casimir energy per unit length of a Sierpinski rectangle or square is $-9 / 71$ times the Casimir energy per unit length of the respective rectangle or square, $\mathcal{E}_{\square}$. The Casimir energy per unit length, $\mathcal{E}_{\Delta}$ and $\mathcal{E}_{\square}$, for the five integrable cylinders are summarized in Table I.
TABLE I. Casimir energy per unit length for cylinders of five cross sections from Ref. [5], referred to as $\mathcal{E}_{\Delta}$ and $\mathcal{E}_{\square}$ in this paper. The cutoff independent finite part is presented. The numbers correspond to the constant $b_{c}$ in Eq. (1) for the respective cross sections, presented here to three significant digits without rounding. The second, third, and fourth columns correspond to the boundary conditions imposed on the fields.

\begin{tabular}{lccc}
\hline \hline Cross section & Dirichlet & Neumann & EM \\
\hline Equilateral triangle & $\frac{0.0237}{a^{2}}$ & $-\frac{0.0613}{a^{2}}$ & $-\frac{0.0375}{a^{2}}$ \\
Hemiequilateral triangle & $\frac{0.0756}{a^{2}}$ & $-\frac{0.0944}{a^{2}}$ & $-\frac{0.0187}{a^{2}}$ \\
Isosceles triangle & $\frac{0.0263}{a^{2}}$ & $-\frac{0.0454}{a^{2}}$ & $-\frac{0.0190}{a^{2}}$ \\
Square & $\frac{0.00483}{a^{2}}$ & $-\frac{0.0429}{a^{2}}$ & $-\frac{0.0381}{a^{2}}$ \\
Rectangle & Refer Ref. [5]. & & \\
\hline \hline
\end{tabular}

contrasted with field theoretic methods of Lifshitz et al. [6] and Schwinger et al. [7] that incorporate both the interior and exterior modes in the evaluation. The Casimir energies for the five geometries of Ref. [5], for empty triangles and rectangles or squares, due to interior modes only, referred to as $\mathcal{E}_{\Delta}$ and $\mathcal{E}_{\square}$ in this paper, are summarized in Table I.

Before we proceed with our discussion, we present the results for the finite part of the Casimir energy for each of the five geometries presented in Fig. 1, which are expressed in terms of $\mathcal{E}_{\Delta}$ and $\mathcal{E}_{\square}$ of Table I. The expression $\mathcal{E}_{s}=$ $-4 \mathcal{E}_{\Delta} / 11$ is universal for all Sierpinski triangles, and $\mathcal{E}_{s}=$ $-9 \mathcal{E}_{\square} / 71$ is universal for all Sierpinski rectangles, and is not restricted to the five geometries of Ref. [5]. However, we often confine the analysis to the five geometries of Ref. [5], because exact expressions were derived for the Casimir energy per unit length for these geometries there.

\section{SIERPINSKI TRIANGLE}

The Sierpinski triangle is self-similar. That is, it consists of copies of the scaled-down versions of itself. Figure 2 shows the Sierpinski triangle of side length $a$, which may be viewed as comprised of three Sierpinski triangles of side length $a / 2$.

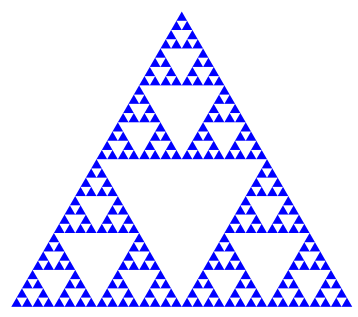

FIG. 2. Sierpinski triangle. The white regions in the interior are triangular cavities, each of which contributes to the Casimir energy per unit length of the Sierpinski triangle. The matter bounding each of the triangles (in blue) is perfectly conducting for the case of electromagnetic fields. 


\section{A. Area and $\delta_{2}$}

Using the self-similarity of a Sierpinski triangle we can write the following recursion relation for the area $A_{s}(a)$ of the cavities inside a Sierpinski triangle:

$$
A_{s}(a)=A_{\Delta}\left(\frac{a}{2}\right)+3 A_{s}\left(\frac{a}{2}\right),
$$

where $A_{\Delta}(a)$ is the area of an equilateral triangle of side length $a$. Using Eq. (7) recursively in itself we obtain the series

$A_{s}(a)=A_{\Delta}\left(\frac{a}{2}\right)+3 A_{\Delta}\left(\frac{a}{2^{2}}\right)+3^{2} A_{\Delta}\left(\frac{a}{2^{3}}\right)+\cdots$.

Then, using the scaling relation of the area of a triangle,

$$
A_{\Delta}\left(\frac{a}{2}\right)=\frac{1}{2^{2}} A_{\Delta}(a),
$$

we obtain the area of the Sierpinski triangle in Fig. 2 to be exactly equal to the area of a triangle. That is,

$$
A_{s}(a)=A_{\Delta}(a) .
$$

Since the area of a triangle $A_{\Delta}(a)$ scales like $a^{2}$, dimensional analysis of Eq. (10) implies that the fractal dimension of the area, defined in Eq. (3), of the Sierpinski triangle is

$$
\delta_{2}=2 \text {. }
$$

\section{B. Perimeter and $\delta_{1}$}

Similarly, the interior perimeter of the cavities inside a Sierpinski triangle satisfies the recursion relation

$$
P_{s}(a)=P_{\Delta}\left(\frac{a}{2}\right)+3 P_{s}\left(\frac{a}{2}\right),
$$

where $P_{\Delta}(a)$ is the interior perimeter of a triangular cavity of side length $a$ and $P_{s}(a)$ is the sum of the interior perimeter of all the individual cavities constituting the Sierpinski triangle. The series constructed from the recursion relation, after using the scaling argument for $P_{\Delta}(a)$, is divergent and leads to

$$
P_{s}(a)=-P_{\Delta}(a)
$$

after using the divergent sum

$$
\frac{1}{2}+\frac{3}{2^{2}}+\frac{3^{2}}{2^{3}}+\cdots=-1,
$$

which can be deduced using the property of self-similarity of the series [8]. Ignoring the counterintuitive nature of a negative perimeter, we learn from Eq. (13) that the fractal dimension for the perimeter, defined in Eq. (3), of the Sierpinski triangle in Fig. 2 is

$$
\delta_{1}=1
$$

because the perimeter of a triangle $P_{\Delta}(a)$ scales like $a$.

\section{Corner angle and $\boldsymbol{\delta}_{\mathbf{0}}$}

The interior corner angles of a Sierpinski triangle satisfy

$$
C_{s}(a)=C_{\Delta}\left(\frac{a}{2}\right)+3 C_{s}\left(\frac{a}{2}\right),
$$

which leads to the series

$C_{s}(a)=C_{\Delta}\left(\frac{a}{2}\right)+3 C_{\Delta}\left(\frac{a}{2^{2}}\right)+3^{2} C_{\Delta}\left(\frac{a}{2^{3}}\right)+\cdots$

The corner term for a triangle is given by [5]

$$
C_{\Delta}(a)=\sum_{i}\left(\frac{\pi}{\alpha_{i}}-\frac{\alpha_{i}}{\pi}\right) \text {, }
$$

where $\alpha_{i}$ are the angles of a triangle, which is independent of the scale $a$. Thus, we can derive

$$
C_{s}(a)=-\frac{1}{2} C_{\Delta}(a)
$$

after using the divergent sum $1+3+3^{2}+\cdots=-1 / 2$. We learn from Eq. (19) that the fractal dimension for the corner angle of the Sierpinski triangle in Fig. 2 is

$$
\delta_{0}=0,
$$

because the corner angle of a triangle $C_{\Delta}(a)$ is scale independent.

\section{Casimir energy and $\boldsymbol{\delta}_{c}$}

Using the decomposition of Casimir energies into singlebody energy and the respective interaction energy between the bodies [9], the Casimir energy per unit length of a Sierpinski triangle $\mathcal{E}_{s}(a)$ can be decomposed as

$$
\mathcal{E}_{s}(a)=\mathcal{E}_{\text {int }}\left(\frac{a}{2}\right)+3 \mathcal{E}_{s}\left(\frac{a}{2}\right),
$$

where $3 \mathcal{E}_{s}(a / 2)$ is the single-body Casimir energy of three Sierpinski triangles of side $a / 2$ in Fig. 2, and $\mathcal{E}_{\text {int }}(a)$ is the interaction energy between the three Sierpinski triangles. Arguably, in general, the interaction energy $\mathcal{E}_{\text {int }}$ depends on both the interior and exterior modes. But the Casimir energies of the cavities we are considering are all due to interior modes. Thus, for consistency, we presume that the interaction energy involves only the interior modes. 


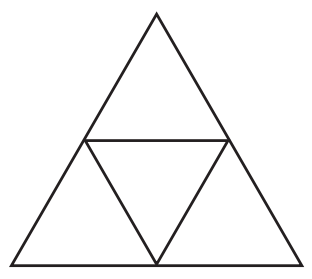

FIG. 3. Four triangles constituting the Sierpinski triangle.

We further justify the consistency of this presumption in the following discussion.

Using Eq. (21) recursively we obtain the series

$\mathcal{E}_{s}(a)=\mathcal{E}_{\text {int }}\left(\frac{a}{2}\right)+3 \mathcal{E}_{\text {int }}\left(\frac{a}{2^{2}}\right)+3^{2} \mathcal{E}_{\text {int }}\left(\frac{a}{2^{3}}\right)+\cdots$.

Thus, the evaluation of the Casimir energy reduces to computing the interaction energy $\mathcal{E}_{\text {int }}$.

Dirichlet boundary conditions require a scalar field to be 0 on the boundary. This restriction essentially separates the physical phenomena on the two sides of the boundary. Thus, the modes and the associated physical phenomena inside Dirichlet cavities are essentially independent of its surroundings. Extending this argument to Sierpinski triangles we learn that the interaction energy between two or more Sierpinski triangles is independent of the internal structure of each of them. We can thus infer that the interaction energy of the three Dirichlet Sierpinski triangles in Fig. 2 is identical to the interaction energy of the three Dirichlet triangles in Fig. 3. We can determine the total energy of the four triangles in Fig. 3 in two independent methods. In the first method we argue that the energy is the sum of the four triangular cavities, $4 \mathcal{E}_{\Delta}\left(\frac{a}{2}\right)$. In the second method we argue that the total energy is the sum of the energies of the three outer triangles, $3 \mathcal{E}_{\Delta}\left(\frac{a}{2}\right)$, plus the interaction energy $\mathcal{E}_{\text {int }}(a / 2)$ between the three triangles. That is,

$$
4 \mathcal{E}_{\Delta}\left(\frac{a}{2}\right)=3 \mathcal{E}_{\Delta}\left(\frac{a}{2}\right)+\mathcal{E}_{\text {int }}\left(\frac{a}{2}\right)
$$

This immediately suggests that the interaction energy of three outer triangles is completely given by the energy of the inner triangle,

$$
\mathcal{E}_{\text {int }}\left(\frac{a}{2}\right)=\mathcal{E}_{\Delta}\left(\frac{a}{2}\right)=2^{2} \mathcal{E}_{\Delta}(a),
$$

where in the second equality we used the fact that the Casimir energy of a triangle $\mathcal{E}_{\Delta}(a)$ scales like the inverse square of length.

Using Eq. (24) in Eq. (22) we derive the Casimir energy of the Dirichlet Sierpinski triangle in terms of the Casimir energy of the equilateral triangle as

$$
\mathcal{E}_{s}(a)=-\frac{4}{11} \mathcal{E}_{\Delta}(a)
$$

using the divergent sum

$$
1+12+12^{2}+\cdots=-\frac{1}{11}
$$

Since the energy per unit length of a triangle $\mathcal{E}_{\Delta}(a)$ scales like inverse length square, Eq. (25) implies that the energy per unit length of the Sierpinski triangle also scales similarly, that is,

$$
\delta_{c}=-2
$$

The Casimir energy per unit length for the Sierpinski extension of a cylinder with arbitrary triangular cross section is also given using Eq. (25). The expression for $\mathcal{E}_{\Delta}$, without the cutoff dependent part, for the three cylinders with triangular cross sections, for which closed-form solutions has been achieved, has been summarized in Table I.

\section{INVERSE SIERPINSKI TRIANGLE}

We define the inverse Sierpinski triangle as the object obtained by swapping the empty space with the perfectly conducting material in the Sierpinski triangle. In Fig. 2 this is obtained by swapping the white color representing empty space with blue color representing perfectly conducting material. See Fig. 4. The outer region in Fig. 4 is unbounded.

The area of the inverse Sierpinski triangle satisfies the relation

$$
A_{s}(a)=3^{n} A_{s}\left(\frac{a}{2^{n}}\right)
$$

for any non-negative integer $n$. Presuming that this area scales like $a^{\delta_{2}}$ we obtain the relation

$$
A_{s}(a)=\frac{3^{n}}{\left(2^{n}\right)^{\delta_{2}}} A_{s}(a)
$$

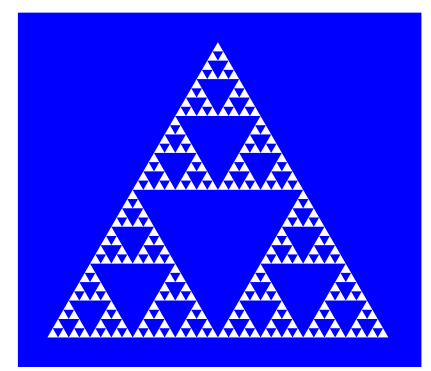

FIG. 4. Inverse Sierpinski triangle. It is obtained from the Sierpinski triangle in Fig. 2 by swapping the empty space with perfectly conducting material there. The blue region extends to infinity. 
for any positive integer $n$. The central idea of nontrivial fractal dimensions stems from Eq. (29) and its solutions. The trivial solution is $A_{s}(a)=0$, which can be envisioned to be a possible scenario by extending Fig. 4 (drawn to five iterations) to infinite iterations. This trivial solution agrees with the notion that the perfectly conducting material in Fig. 4 fills all space in this limit. But Eq. (29) also admits a nontrivial solution, namely

$$
1=\left(\frac{3}{2^{\delta_{2}}}\right)^{n},
$$

for any positive integer $n$, which immediately implies that

$$
\delta_{2}=\frac{\ln 3}{\ln 2} \sim 1.58496 .
$$

The nontrivial solution here is probably a consequence of the nontrivial solution of a divergent series as a regularized sum, which has the trivial solution to be infinity [8].

The perimeter also satisfies the relation in Eq. (28) with the areas $A$ now replaced with perimeters $P$. The corner angles also satisfy Eq. (28). Further, the Casimir energy per unit length also satisfies Eq. (28). Thus, we learn that

$$
\delta_{c}=\delta_{2}=\delta_{1}=\delta_{0}=\frac{\ln 3}{\ln 2},
$$

unless we confine to the trivial solution that area, perimeter, angles, and the energy per unit length are all 0 .

The fact that the fractal dimensions of all the relevant physical quantities, the area, the perimeter, the corner angle, and the Casimir energy per unit length, scale the same way, in conjunction with the generalized Berry conjecture of Eq. (4) implies that there are no divergent terms in the Casimir energy per unit length of the inverse Sierpinski triangle. That is,

$$
\begin{aligned}
\mathcal{E}(a) & =b_{c} a^{\delta_{c}}+\lim _{\tau \rightarrow 0} \tau^{\delta_{c}}\left[b_{2}^{\prime}\left(\frac{a}{\tau}\right)^{\delta_{c}}+b_{1}^{\prime}\left(\frac{a}{\tau}\right)^{\delta_{c}}+b_{0}^{\prime}\left(\frac{a}{\tau}\right)^{\delta_{c}}\right] \\
& =\left(b_{c}+b_{2}^{\prime}+b_{1}^{\prime}+b_{0}^{\prime}\right) a^{\delta_{c}}
\end{aligned}
$$

where $b_{2}^{\prime}, b_{1}^{\prime}$, and $b_{0}^{\prime}$ are redefined constants with respect to the constants $b_{2}, b_{1}$, and $b_{0}$, in Eq. (4), to accommodate numerical constants inside $A(x), P(x)$, and $C(x)$, respectively.

\section{SIERPINSKI CARPET}

The Sierpinski carpet, or a Sierpinski rectangle or square, is the rectangular version of a Sierpinski triangle; see the rectangle and square version in Fig. 1. A Sierpinski carpet satisfies the energy decomposition

$$
\mathcal{E}_{c}(a)=\mathcal{E}_{\text {int }}\left(\frac{a}{3}\right)+8 \mathcal{E}_{c}\left(\frac{a}{3}\right),
$$

which leads to $\mathcal{E}_{c}(a)=-\mathcal{E}_{\text {int }}(a / 3) / 71$. Again, to be consistent with the fact that we are including only the interior modes, the interaction energy $\mathcal{E}_{\text {int }}(a / 3)$ is equal to the Casimir energy of a square enclosed between the eight surrounding squares. Thus, we have

$$
\mathcal{E}_{\text {int }}\left(\frac{a}{3}\right)=\mathcal{E}_{\square}\left(\frac{a}{3}\right)=3^{2} \mathcal{E}_{\square}(a),
$$

where $\mathcal{E}_{\square}(a)$ is the Casimir energy per unit length of a square of side length $a$. Hence, the Casimir energy of the Sierpinski square is evaluated as

$$
\mathcal{E}_{c}(a)=-\frac{9}{71} \mathcal{E}_{\square}(a) .
$$

The calculation for the Sierpinski rectangle goes through the same procedure, because the length and width have a fixed aspect ratio. The Casimir energy per unit length for the Sierpinski extension of a cylinder with arbitrary rectangular cross section is also given using Eq. (36). The expression for $\mathcal{E}_{\square}$, without the cutoff dependent part, for the cylinders with rectangular cross sections, for which closed-form solutions have been found, is summarized in Table I.

The vacuum energy of the inverse Sierpinski square or rectangle satisfies the relation

$$
\mathcal{E}_{c}(a)=8^{n} \mathcal{E}_{c}\left(\frac{a}{3^{n}}\right)
$$

which implies $\delta_{c}=\ln 8 / \ln 3$. Since the area, the perimeter, and the corner angles satisfy the same relation, of Eq. (37), we also learn that $\delta_{c}=\delta_{2}=\delta_{1}=\delta_{0}$. Thus, using the same arguments used to derive Eq. (33), the Casimir energy per unit length of the inverse Sierpinski rectangle or square does not have divergent terms.

We can also extend our discussion to non-Sierpinski fractals. The Vicsek fractal, illustrated in Fig. 5, is obtained by starting from a square, dividing it into nine equal squares of one-third side, and removing four of them. The inverse Vicsek fractal is obtained by swapping the empty space

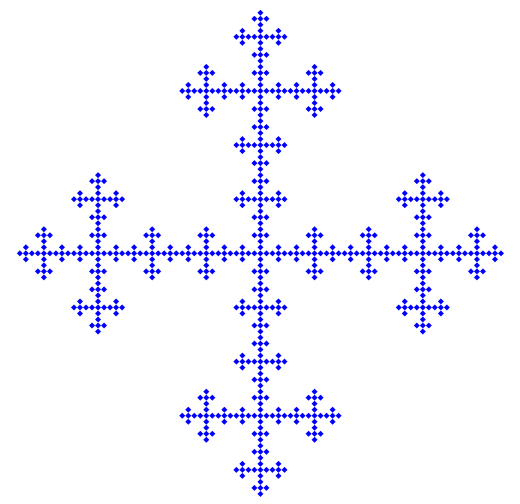

FIG. 5. Vicsek fractal. 
with perfectly conducting material, such that the Casimir energy is given in terms of the energies of the individual cavities. The vacuum energy of the inverse Vicsek fractal satisfies the relation

$$
\mathcal{E}_{v}(a)=5^{n} \mathcal{E}_{v}\left(\frac{a}{3^{n}}\right),
$$

and the area, the perimeter, and the corner angles of the Vicsek fractal also satisfy the same relation. Like in the case of the inverse Sierpinski triangle, Eq. (38) for energy and the corresponding relations admits the nontrivial solution

$$
\delta_{c}=\delta_{2}=\delta_{1}=\delta_{0}=\frac{\ln 5}{\ln 3} \sim 1.46497 .
$$

Thus, using the generalized Berry conjecture of Eq. (4) we can conclude that the Casimir energy per unit length of the inverse Vicsek fractal will not have divergent terms.

\section{DISCUSSION}

We list the fractal dimensions of the geometries discussed here in Table II. For the Sierpinski triangle and the Sierpinski carpet we have shown that the Berry conjecture holds true. But these are relatively trivial cases because the fractal dimensions for these cases are equal to the respective topological dimensions. For the inverse Sierpinski triangle, the inverse Sierpinski carpet, and the inverse Vicsek fractal, even though we encounter nontrivial fractal dimensions, these dimensions (for area, perimeter, corner angle, and Casimir energy) turn out to be all equal. This, in turn, remarkably, gives no room for the divergent terms in the Weyl expansion. Thus, in the absence of the divergent terms, even though the Berry's conjecture holds in principle, we cannot conclude this to be a nontrivial verification of the conjecture.

Berry's conjecture was probably motivated for fractals like the Koch snowflake [10], a simply connected domain,

TABLE II. Fractal dimensions of the area of cavities, $\delta_{2}$, of the perimeter of cavities, $\delta_{1}$, of the corner angles of cavities, $\delta_{0}$, and of the Casimir energy per unit length, $\delta_{c}$, for a few geometries. We note that $\ln 3 / \ln 2 \sim 1.58496, \quad \ln 8 / \ln 3 \sim 1.89279$, $\ln 5 / \ln 3 \sim 1.46497$, and $\ln 4 / \ln 3 \sim 1.26186$. The question mark indicates the value that remains to be calculated, the Casimir energy per unit length of a cylinder with the cross section of the shape of a Koch snowflake.

\begin{tabular}{lrrrc}
\hline \hline Geometry & $\delta_{2}$ & $\delta_{1}$ & $\delta_{0}$ & $\delta_{c}$ \\
\hline Sierpinski triangle & 2 & 1 & 0 & -2 \\
Inverse Sierpinski triangle & $\frac{\ln 3}{\ln 2}$ & $\frac{\ln 3}{\ln 2}$ & $\frac{\ln 3}{\ln 2}$ & $\frac{\ln 3}{\ln 2}$ \\
Sierpinski carpet & 2 & 1 & 0 & -2 \\
Inverse Sierpinski carpet & $\frac{\ln 8}{\ln 3}$ & $\frac{\ln 8}{\ln 3}$ & $\frac{\ln 8}{\ln 3}$ & $\frac{\ln 8}{\ln 3}$ \\
Inverse Vicsek fractal & $\frac{\ln 5}{\ln 3}$ & $\frac{\ln 5}{\ln 3}$ & $\frac{\ln 5}{\ln 3}$ & $\frac{\ln 5}{\ln 3}$ \\
Koch snowflake & 2 & $\frac{\ln 4}{\ln 3}$ & 0 & $?$ \\
\hline \hline
\end{tabular}

in which the perimeter encloses a single continuously connected region. The area and the corner angles of a Koch curve scale normally, but the perimeter scales like a fractal with a fractal dimension of $\ln 4 / \ln 3 \sim 1.26$. This difference in the scaling behavior between area and perimeter, we believe, is suitable for studying the Berry conjecture. The methods presented here do not seem to yield the Casimir energy of a Koch snowflake.

The perimeter of a Koch snowflake scales like $P_{k}(a)=4^{n} P_{k}\left(a / 3^{n}\right)$, which implies that for a Koch snowflake $\delta_{1}=\ln 4 / \ln 3$. In contrast, the area of a Koch snowflake $A_{k}(a)$ is given by $A_{k}(a)=A_{\Delta}(a)+3 A_{k}^{\prime}(a)$, expressed in terms of a reduced area $A_{k}^{\prime}(a)$ that satisfies the recursion relation $A_{k}^{\prime}(a)=A_{\Delta}(a / 3)+4 A_{k}^{\prime}(a / 3)$, which leads to $A_{k}(a)=8 A_{\Delta}(a) / 5$ and implies that for a Koch snowflake $\delta_{2}=2$. Further, the corner angles $c_{k}(a)$ of a Koch snowflake satisfy $c_{k}(a)=6 \pi+4 c_{k}(a / 3)$, which leads to $c_{k}(a)=-2 \pi$. Thus, for a Koch snowflake $\delta_{0}=0$.

It seems that it would be more appropriate to analyze the Berry conjecture for a Koch snowflake, because of the fact that its perimeter scales like a fractal while its area scales normally. In the Sierpinski triangle, and in the inverse Sierpinski triangle, the cavities are compartmentalized. These geometries are not simply connected. This feature in conjunction with the idea of self-similarity was the key to our evaluation of the Casimir energies of the fractal geometries considered here. Without the feature of compartmentalization we are unable to calculate the Casimir energy of a Koch snowflake as yet. Thus, we are unable to analyze the Berry conjecture for a Koch snowflake.

In the literature, the Berry conjecture was formulated and has been studied for the distribution of the modes of a cavity. Casimir energy is directly related to the sum of all modes of a cavity, and gets divergent contributions dictated by the distribution of the modes for large frequencies. There seems to be an extensive literature on the Weyl law and the associated Berry conjecture. We found the review in Ref. [11] very resourceful. Nevertheless, to our knowledge, Berry's conjecture has not been addressed in the context of Casimir energies before. The only exception seems to be the discussion in Ref. [12] where fractal geometries are discussed in the context of heat kernels, which is a powerful technique used to extract divergent terms in Casimir energy. Our method exploits the formalism of Green's functions that was described in the context of self-similar plates in Ref. [3], and its correspondence with the heat kernel method is well known in the community. In Ref. [5], the method of mode summation was used, so the connection with heat-kernel methods should not be so remote. In our understanding, there is no direct overlap between the discussions we have presented here with those in Ref. [12], but the connections should be worth pursuing.

In the studies on distribution of modes, without concerning Casimir energy, it has been argued in Ref. [13] that the dimensions of the regions and surfaces should be 
interpreted as the Minkowski-Bouligand dimension instead of the Hausdorff-Besicovitch dimension as originally proposed by Berry. This was further promoted in Ref. [14]. Counterexamples involving domains that are not simply connected were presented in Ref. [15], but it has been suggested that the conjecture is expected to hold for simply connected fractals like the Koch snowflake. These conclusions seem to be in agreement with our results here in the context of Casimir energies. No doubt, Berry's conjecture needs to be explored further.

\section{ACKNOWLEDGMENTS}

We thank Kimball A. Milton for discussions, and for directing our attention to the Casimir energy calculations using interior modes alone that was essential for this work. We thank Mathias Boström for discussions. We acknowledge support from the Research Council of Norway (Project No. 250346). I. C. P. acknowledges support from Centro Universitario de la Defensa (Grant No. CUD201512) and DGA-FSE (Grant No. 2015-E24/2).
[1] H. Weyl, Ueber die asymptotische Verteilung der Eigenwerte, Nachr. Ges. Wiss. Göttingen, Math.-Phys. K1. 1911, 110 (1911).

[2] M. V. Berry, in Structural Stability in Physics, edited by W. Güttinger and H. Eikemeier (Spinger, New York, 1979), p. 51.

[3] K. V. Shajesh, I. Brevik, I. Cavero-Peláez, and P. Parashar, Casimir energies of self-similar plate configurations, Phys. Rev. D 94, 065003 (2016).

[4] D. R. Hofstadter, Energy levels and wave functions of Bloch electrons in rational and irrational magnetic fields, Phys. Rev. B 14, 2239 (1976).

[5] E. K. Abalo, K. A. Milton, and L. Kaplan, Casimir energies of cylinders: Universal function, Phys. Rev. D 82, 125007 (2010).

[6] I. E. Dzyaloshinskii, E. M. Lifshitz, and L. P. Pitaevskii, The general theory of van der Waals forces, Adv. Phys. 10, 165 (1961).

[7] J. Schwinger, L. L. DeRaad, Jr., and K. A. Milton, Casimir effect in dielectrics, Ann. Phys. (N.Y.) 115, 1 (1978).

[8] G. H. Hardy, Divergent Series (Clarendon, Oxford, 1956).
[9] K. V. Shajesh and M. Schaden, Many-body contributions to Green's functions and Casimir energies, Phys. Rev. D 83, 125032 (2011).

[10] B. B. Mandelbrot, The Fractal Geometry of Nature (W. H. Freeman and Company, New York, 1977).

[11] W. Arendt, R. Nittka, W. Peter, and F. Steiner, in Mathematical Analysis of Evolution, Information, and Complexity (Wiley-VCH Verlag GmbH \& Co. KGaA, Weinheim, Germany, 2009), p. 1.

[12] G. V. Dunne, Heat kernels and zeta functions on fractals, J. Phys. A 45, 374016 (2012).

[13] J. Brossard and R. Carmona, Can one hear the dimension of a fractal?, Commun. Math. Phys. 104, 103 (1986).

[14] M. L. Lapidus and J. Fleckinger, Tambour fractal: Vers une résolution de la conjecture de Weyl-Berry pour les valeurs propres du Laplacien [Fractal drum: Towards a resolution of the Weyl-Berry conjecture for the eigenvalues of the Laplacian], C. R. Acad. Sci. Paris, Sér. I Math. 306, 171 (1988).

[15] M. L. Lapidus and C. Pomerance, Counterexamples to the modified Weyl-Berry conjecture on fractal drums, Math. Proc. Cambridge Philos. Soc. 119, 167 (1996). 